PROCEEDINGS OF THE

AMERICAN MATHEMATICAL SOCIETY

Volume 127, Number 3, March 1999, Pages 709-714

S 0002-9939(99)04562-1

\title{
LIE ALGEBRAS OF COHOMOLOGICAL CODIMENSION ONE
}

\author{
GRANT F. ARMSTRONG, GRANT CAIRNS, AND GUNKY KIM
}

(Communicated by Roe Goodman)

\begin{abstract}
We show that if $\mathfrak{g}$ is a finite dimensional real Lie algebra, then $\mathfrak{g}$ has cohomological dimension $c d(\mathfrak{g})=\operatorname{dim}(\mathfrak{g})-1$ if and only if $\mathfrak{g}$ is a unimodular extension of the two-dimensional non-Abelian Lie algebra $\mathfrak{a} \mathfrak{f}$.
\end{abstract}

\section{INTRODUCTION}

Throughout this paper, $\mathfrak{g}$ denotes a finite dimensional real Lie algebra. The cohomological dimension of $\mathfrak{g}$ is the largest integer $c d(\mathfrak{g})$ such that $H^{c d}(\mathfrak{g})(\mathfrak{g}, \mathbb{R}) \neq 0$, where $H^{*}(\mathfrak{g}, \mathbb{R})$ denotes the cohomology of $\mathfrak{g}$ with trivial coefficients. It is well known that $c d(\mathfrak{g})=\operatorname{dim}(\mathfrak{g})$ if and only if $\mathfrak{g}$ is unimodular [1]; that is, if the adjoint map $a d(x): y \mapsto[x, y]$ has zero trace for all $x \in \mathfrak{g}$. So in the space of Lie algebras of any given dimension, the subset of Lie algebras with $\operatorname{cd}(\mathfrak{g})<\operatorname{dim}(\mathfrak{g})$ constitutes a large open set. For example, in dimension 3 there are infinitely many nonisomorphic real Lie algebras, but only 6 isomorphism classes having $\operatorname{cd}(\mathfrak{g})=\operatorname{dim}(\mathfrak{g})$ (see [2]). Interestingly, the algebras with $\operatorname{cd}(\mathfrak{g})=\operatorname{dim}(\mathfrak{g})-1$ are also quite rare. The simplest Lie algebra with $c d(\mathfrak{a} \mathfrak{f} \mathfrak{f})=\operatorname{dim}(\mathfrak{g})-1$ is the 2-dimensional affine Lie algebra $\mathfrak{a f f}=\langle x, y:[x, y]=y\rangle$.

Definition. An extension $\mathfrak{a} \hookrightarrow \mathfrak{g} \rightarrow \mathfrak{b}$, of a Lie algebra $\mathfrak{b}$ by a Lie algebra $\mathfrak{a}$, is said to be unimodular if $\left.a d(x)\right|_{\mathfrak{a}}$ has zero trace for all $x \in \mathfrak{g}$.

Theorem 1.1. $\operatorname{cd}(\mathfrak{g})=\operatorname{dim}(\mathfrak{g})-1$ if and only if $\mathfrak{g}$ is a unimodular extension of $\mathfrak{a f f}$.

It follows from Theorem 1.1 that the only Lie algebra of dimension 3 with $c d(\mathfrak{g})=$ $\operatorname{dim}(\mathfrak{g})-1$ is $\mathbb{R} \oplus \mathfrak{a f f f}$. In the classification of 3-dimensional Lie algebras, $\mathbb{R} \oplus \mathfrak{a} \mathfrak{f} \mathfrak{f}$ is the algebra with $\chi=\infty$, where $\chi$ is the Tasaki-Umehara invariant [3].

The paper is organized as follows. In Section 2 we establish some duality results which we require in the proof of Theorem 1.1. In Section 3 we give some elementary results for unimodular extensions. Theorem 1.1 is then proved in Section 4. The paper finishes with some remarks, in Section 5.

Let us introduce some notation and basic definitions. For convenience, we write $\operatorname{dim}(\mathfrak{g})=n$. When there is no risk of confusion, we will use the notation $\Lambda^{i}$ to denote the $i$-th exterior product $\Lambda^{i} \mathfrak{g}^{*}$ of the dual space $\mathfrak{g}^{*}$. We denote $\bigoplus_{i=0}^{n} \Lambda^{i}$ by $\Lambda$. Recall that a linear map $\phi: \Lambda \rightarrow \Lambda$ is a derivation of degree $k$ if $\phi(\alpha \wedge \beta)=$ $(\phi \alpha) \wedge \beta+(-1)^{k \cdot i} \alpha \wedge(\phi \beta)$, for all $\alpha \in \Lambda^{i}, \beta \in \Lambda$. The differential $d$ in $\Lambda$ is

Received by the editors May 13, 1997 and, in revised form, July 7, 1997.

1991 Mathematics Subject Classification. Primary 17B56.

Key words and phrases. Lie algebra, cohomology, cohomological dimension.

(C)1999 American Mathematical Society 
the derivation of degree 1 whose restriction to $\Lambda^{1}$ is the dual of the Lie product [, ] : $\Lambda^{2} \mathfrak{g} \rightarrow \Lambda^{1} \mathfrak{g}$. The cohomology $H^{*}(\Lambda, \mathbb{R})$ of $\mathfrak{g}$ is the cohomology of $d$. The canonical $1-$ form $\gamma \in \Lambda^{1}$ is defined by $\gamma(x)=\operatorname{tr}(\operatorname{ad}(x))$ for all $x \in \mathfrak{g}$.

\section{TWisted DUALity}

Let $D: \Lambda \rightarrow \Lambda$ be defined by $D(\alpha)=d \alpha-\gamma \wedge \alpha$ for all $\alpha \in \Lambda$. Note that $\gamma$ is $d$-closed and for all $\alpha \in \Lambda^{n-1}$, one has $d \alpha=\gamma \wedge \alpha$. So $D \alpha=0$ for all $\alpha \in \Lambda^{n-1}$. Notice that $D$ is not a derivation, but instead one has

$$
D(\alpha \wedge \beta)=(D \alpha) \wedge \beta+(-1)^{i} \alpha \wedge(d \beta)=(d \alpha) \wedge \beta+(-1)^{i} \alpha \wedge(D \beta),
$$

for all $\alpha \in \Lambda^{i}, \beta \in \Lambda$. One also sees easily that $D^{2}=0$ and so we can define the twisted cohomology groups $H_{D}^{*}(\mathfrak{g}, \mathbb{R})=\operatorname{ker} D / \operatorname{Im} D$. The main purpose of this present section is to prove:

Theorem 2.1 (Poincaré duality for Lie algebras). For all $i \in\{0, \ldots, n\}$, one has $H^{i}(\mathfrak{g}, \mathbb{R}) \cong H_{D}^{n-i}(\mathfrak{g}, \mathbb{R})$.

To prove this result we first recall the Hodge star-operator $*: \Lambda \rightarrow \Lambda$. Choose a basis $\left\{x_{1}, \ldots, x_{n}\right\}$ for $\mathfrak{g}$ and consider the dual basis $\left\{\alpha_{1}, \ldots, \alpha_{n}\right\}$ for $\Lambda^{1}$. Then for each $i \in\{0, \ldots, n\}$, set

$$
*\left(\alpha_{\sigma(1)} \wedge \cdots \wedge \alpha_{\sigma(i)}\right)=(-1)^{\operatorname{sgn}(\sigma)} \alpha_{\sigma(i+1)} \wedge \cdots \wedge \alpha_{\sigma(n)},
$$

for every permutation $\sigma \in S_{n}$. Then $*$ extends to a unique linear isomorphism $*: \Lambda^{i} \rightarrow \Lambda^{n-i}$ and $* *=(-1)^{i(n-i)}$. Notice that the bilinear map \langle\rangle$: \Lambda \times \Lambda \rightarrow \mathbb{R}$ defined by $\langle\alpha, \beta\rangle=*(\alpha \wedge * \beta)$ is just the inner product on $\Lambda$ with respect to which the basis elements of the form $\alpha_{j_{1}} \wedge \cdots \wedge \alpha_{j_{i}}$ are orthonormal. Next define $\partial: \Lambda \rightarrow \Lambda$ by defining it on $\Lambda^{i}$ to be $\partial=(-1)^{n(i+1)+1} * D *$, and set $\Delta=\partial d+d \partial$. One has:

Lemma 2.2. (a) $\partial$ is the adjoint of $d$; that is, $\langle d \alpha, \beta\rangle=\langle\alpha, \partial \beta\rangle$, for all $\alpha, \beta \in \Lambda$.

(b) $\Delta$ is self-adjoint; that is, $\langle\Delta \alpha, \beta\rangle=\langle\alpha, \Delta \beta\rangle$, for all $\alpha, \beta \in \Lambda$.

(c) $\operatorname{ker} \Delta=\operatorname{ker} d \cap \operatorname{ker} \partial$.

Proof. (a) Let $i \in\{0, \ldots, n\}$ and let $\alpha \in \Lambda^{i}, \beta \in \Lambda^{i+1}$. We are required to show that $*(d \alpha \wedge * \beta)=*(\alpha \wedge * \partial \beta)$ or equivalently $d \alpha \wedge * \beta=\alpha \wedge * \partial \beta$. Since $\alpha \wedge * \beta \in \Lambda^{n-1}$, we have $D(\alpha \wedge * \beta)=0$ and so by $(\dagger)$,

$$
\begin{aligned}
\alpha \wedge * \partial \beta=\alpha \wedge *(-1)^{n(i+2)+1} * D * \beta & =(-1)^{n(i+2)+1+i(n-i)} \alpha \wedge D * \beta \\
& =-(-1)^{i} \alpha \wedge D * \beta=d \alpha \wedge * \beta .
\end{aligned}
$$

(b) By part (a),

$$
\begin{aligned}
\langle\Delta \alpha, \beta\rangle & =\langle d \partial \alpha, \beta\rangle+\langle\partial d \alpha, \beta\rangle=\langle\partial \alpha, \partial \beta\rangle+\langle d \alpha, d \beta\rangle \\
& =\langle\alpha, d \partial \beta\rangle+\langle\alpha, \partial d \beta\rangle=\langle\alpha, \Delta \beta\rangle .
\end{aligned}
$$

(c) Clearly ker $d \cap \operatorname{ker} \partial \subset \operatorname{ker} \Delta$. Conversely, let $\alpha \in \operatorname{ker} \Delta$. Then by part (a),

$$
0=\langle\Delta \alpha, \alpha\rangle=\langle d \partial \alpha+\partial d \alpha, \alpha\rangle=\langle\partial \alpha, \partial \alpha\rangle+\langle d \alpha, d \alpha\rangle,
$$

which implies that $\partial \alpha=0$ and $d \alpha=0$, as required.

Theorem 2.3 (The Hodge decomposition theorem for Lie algebras). (a) $\Lambda=$ $\operatorname{Im} \Delta \oplus \operatorname{ker} \Delta$.

(b) $\Lambda=\operatorname{Im} d \oplus \operatorname{Im} \partial \oplus \operatorname{ker} \Delta$.

(c) $\operatorname{ker} d=\operatorname{Im} d \oplus \operatorname{ker} \Delta$. 
Proof. (a) First, we claim that $\operatorname{Im} \Delta \cap \operatorname{ker} \Delta=0$. To show this, suppose that $\beta \in \operatorname{Im} \Delta \cap \operatorname{ker} \Delta$. Then $\beta=\Delta \alpha$ for some $\alpha$ and $0=\Delta \beta=\Delta^{2} \alpha$. Hence by Lemma 2.2(b),

$$
0=\left\langle\Delta^{2} \alpha, \alpha\right\rangle=\langle\Delta \alpha, \Delta \alpha\rangle .
$$

So $\beta=\Delta \alpha=0$. Thus $\operatorname{Im} \Delta \cap \operatorname{ker} \Delta=0$ and so $\Lambda=\operatorname{Im} \Delta \oplus \operatorname{ker} \Delta$ for dimension reasons.

(b) Note that $\operatorname{Im} \Delta \subset \operatorname{Im} d+\operatorname{Im} \partial$, by the definition of $\Delta$. So, by (a) it suffices to establish the following 3 conditions: (i) $\operatorname{Im} d \cap \operatorname{Im} \partial=0$, (ii) $\operatorname{Im} d \cap \operatorname{ker} \Delta=0$, (iii) $\operatorname{Im} \partial \cap \operatorname{ker} \Delta=0$.

(i) Suppose that $\alpha=d \beta$ and $\alpha=\partial \gamma$ for some $\beta$ and $\gamma$. Then by Lemma 2.2(a), $\langle\alpha, \alpha\rangle=\langle d \beta, \partial \gamma\rangle=\left\langle d^{2} \beta, \gamma\right\rangle=0$. Hence $\alpha=0$ as required.

(ii) Suppose that $\alpha=d \beta$ for some $\beta$ and that $\Delta \alpha=0$. Then by Lemma 2.2(c), $\partial \alpha=0$. So $0=\langle\partial \alpha, \beta\rangle=\langle\partial d \beta, \beta\rangle=\langle d \beta, d \beta\rangle$, which gives $d \beta=0$. Hence $\alpha=0$ as required.

(iii) Suppose that $\alpha=\partial \beta$ for some $\beta$ and that $\Delta \alpha=0$. Then by Lemma 2.2(c), $d \alpha=0$. So $0=\langle d \alpha, \beta\rangle=\langle d \partial \beta, \beta\rangle=\langle\partial \beta, \partial \beta\rangle$, which gives $\partial \beta=0$. Hence $\alpha=0$ as required. This establishes (b).

(c) By part (b), since $\operatorname{Im} d \oplus \operatorname{ker} \Delta \subset \operatorname{ker} d$, it suffices to show that $\operatorname{ker} d \cap \operatorname{Im} \partial=$ 0 . Suppose that $\alpha \in \operatorname{ker} d$ and that $\alpha=\partial \beta$ for some $\beta \in \Lambda$. Then

$$
0=\langle d \alpha, \beta\rangle=\langle\alpha, \partial \beta\rangle=\langle\partial \beta, \partial \beta\rangle .
$$

Hence $\alpha=0$, as required.

We now present a dual version of Theorem 2.3. First, define $\delta: \Lambda \rightarrow \Lambda$ by defining it on $\Lambda^{i}$ to be $\delta=(-1)^{n(i+1)+1} * d *$, and set $\underline{\Delta}=\delta D+D \delta$. Then analogous to Lemma 2.2, one finds in the same way that:

Lemma 2.4. $\delta$ is the adjoint of $D, \underline{\Delta}$ is self-adjoint and $\operatorname{ker} \Delta=\operatorname{ker} D \cap \operatorname{ker} \delta$.

Just as in the proof of Theorem 2.3, Lemma 2.4 can then be used to establish:

Theorem 2.5. $\Lambda=\operatorname{Im} \underline{\Delta} \oplus \operatorname{ker} \underline{\Delta}=\operatorname{Im} D \oplus \operatorname{Im} \delta \oplus \operatorname{ker} \underline{\Delta}$ and $\operatorname{ker} D=\operatorname{Im} D \oplus \operatorname{ker} \underline{\Delta}$.

We can now prove Theorem 2.1. By Theorem 2.3,

$$
H^{*}(\mathfrak{g}, \mathbb{R})=\operatorname{ker} d / \operatorname{Im} d=(\operatorname{Im} d \oplus \operatorname{ker} \Delta) / \operatorname{Im} d \cong \operatorname{ker} \Delta .
$$

Similarly, by Theorem 2.5 , one has $H_{D}^{*}(\mathfrak{g}, \mathbb{R}) \cong$ ker $\underline{\Delta}$. Theorem 2.1 then follows immediately from the fact that $*: \operatorname{ker} \Delta \rightarrow \operatorname{ker} \underline{\Delta}$ is an isomorphism.

\section{UNIMODULAR EXTENSIONS}

It is well known that if $\alpha \in \Lambda^{1}$ is $d$-closed, then $\operatorname{ker} \alpha$ is an ideal. Let $\mathfrak{a}=$ $\operatorname{ker} \alpha \cap \operatorname{ker} \gamma$. We have:

Lemma 3.1. If $\alpha \in \Lambda^{1}$ is D-closed, then $\mathfrak{a}$ is an ideal.

Proof. Suppose that $\alpha$ is $D$-closed. Let $x \in \mathfrak{g}$ and $y \in \mathfrak{a}$. Since $\operatorname{ker} \gamma$ is an ideal, we have $[x, y] \in \operatorname{ker} \gamma$. Since $D \alpha=0$ and $y \in \mathfrak{a}$, one has

$$
\alpha([x, y])=d \alpha(x, y)=\gamma \wedge \alpha(x, y)=0 .
$$

So $[x, y] \in \mathfrak{a}$. Hence $\mathfrak{a}$ is an ideal. 
Lemma 3.2. If $\alpha \in \Lambda^{1}$ and $\alpha \neq 0$, then $\alpha$ is $D$-exact if and only if $\operatorname{ker} \alpha=\operatorname{ker} \gamma$.

Proof. Indeed, $\alpha$ is $D$-exact if and only if $\alpha=k \gamma$ for some $k \in \mathbb{R}$.

Now consider an extension $\mathfrak{a} \hookrightarrow \mathfrak{g} \rightarrow \mathfrak{g} / \mathfrak{a}$. The natural projection $\phi: \mathfrak{g} \rightarrow \mathfrak{g} / \mathfrak{a}$ induces a "pull-back" map $\phi^{*}: \Lambda(\mathfrak{g} / \mathfrak{a})^{*} \rightarrow \Lambda \mathfrak{g}^{*}$ : if $\alpha \in \Lambda^{i}(\mathfrak{g} / \mathfrak{a})^{*}$, then the $i$-form $\phi^{*} \alpha$ is defined by

$$
\left(\phi^{*} \alpha\right)\left(x_{1}, \ldots, x_{i}\right)=\alpha\left(\phi x_{1}, \ldots, \phi x_{i}\right)
$$

for all $x_{1}, \ldots, x_{i} \in \mathfrak{g}$. It is well known that $\phi^{*}$ commutes with $d$ and thus induces a homomorphism $\phi^{\#}: H^{*}(\mathfrak{g} / \mathfrak{a}, \mathbb{R}) \rightarrow H^{*}(\mathfrak{g}, \mathbb{R})$.

Lemma 3.3. If $\mathfrak{a} \hookrightarrow \mathfrak{g} \rightarrow \mathfrak{g} / \mathfrak{a}$ is a unimodular extension, then $\phi^{*}$ commutes with $D$.

Proof. Let the canonical 1-forms of $\mathfrak{g}$ and $\mathfrak{g} / \mathfrak{a}$ be denoted $\gamma_{\mathfrak{g}}$ and $\gamma_{\mathfrak{g} / \mathfrak{a}}$, respectively. Since the extension is unimodular, one has $\gamma_{\mathfrak{g}}=\phi^{*}\left(\gamma_{\mathfrak{g} / \mathfrak{a}}\right)$. So

$$
D\left(\phi^{*} \alpha\right)=d\left(\phi^{*} \alpha\right)-\gamma_{\mathfrak{g}} \wedge\left(\phi^{*} \alpha\right)=\phi^{*}(d \alpha)-\phi^{*}\left(\gamma_{\mathfrak{g} / \mathfrak{a}} \wedge \alpha\right)=\phi^{*}(D \alpha) .
$$

By the above lemma, $\phi^{*}$ induces a homomorphism $\phi^{\#}: H_{D}^{*}(\mathfrak{g} / \mathfrak{a}, \mathbb{R}) \rightarrow H_{D}^{*}(\mathfrak{g}, \mathbb{R})$.

Lemma 3.4. If $\mathfrak{a} \hookrightarrow \mathfrak{g} \rightarrow \mathfrak{g} / \mathfrak{a}$ is a unimodular extension, then the induced map $\phi^{\#}: H_{D}^{1}(\mathfrak{g} / \mathfrak{a}, \mathbb{R}) \rightarrow H_{D}^{1}(\mathfrak{g}, \mathbb{R})$ is injective.

Proof. Suppose that $\alpha \in \Lambda^{1}(\mathfrak{g} / \mathfrak{a})^{*}$ and that $\phi^{*} \alpha$ is $D$-exact. Then $\phi^{*} \alpha=k \gamma_{\mathfrak{g}}$ for some $k \in \mathbb{R}$. So $\phi^{*}\left(\alpha-k \gamma_{\mathfrak{g} / \mathfrak{a}}\right)=0$ and hence $\alpha=k \gamma_{\mathfrak{g} / \mathfrak{a}}$ and $\alpha$ is $D$-exact.

\section{Proof of Theorem 1.1}

First suppose that there exists a unimodular extension $\mathfrak{a} \hookrightarrow \mathfrak{g} \rightarrow \mathfrak{a f f}$. Notice that $\mathfrak{g}$ can't be unimodular, since $\mathfrak{a f f}$ isn't. Hence $c d(\mathfrak{g})<n$. Since $H^{1}(\mathfrak{a} \mathfrak{f} \mathfrak{f}, \mathbb{R}) \neq 0$ one has by Poincaré duality that $H_{D}^{1}(\mathfrak{a} \mathfrak{f} \mathfrak{f}, \mathbb{R}) \neq 0$. Hence, by Lemma $3.4, H_{D}^{1}(\mathfrak{g}, \mathbb{R}) \neq 0$. So by Poincaré duality again, $H^{n-1}(\mathfrak{g}, \mathbb{R}) \neq 0$ and hence $c d(\mathfrak{g})=n-1$.

Conversely, suppose that $c d(\mathfrak{g})=n-1$. It follows by Poincaré duality that there exists a $D$-closed 1 -form $\alpha \in \Lambda$ which is not $D$-exact. Let $\mathfrak{a}=\operatorname{ker} \alpha \cap \operatorname{ker} \gamma$. By Lemma 3.1, $\mathfrak{a}$ is an ideal, and by Lemma 3.2, $\mathfrak{a}$ has codimension 2. It remains to show that $\mathfrak{g} / \mathfrak{a} \cong \mathfrak{a} \mathfrak{f} \mathfrak{f}$ and that the extension $\mathfrak{a} \hookrightarrow \mathfrak{g} \rightarrow \mathfrak{a} \mathfrak{f f}$ is unimodular. Choose elements $x, y \in \mathfrak{g}$ such that $\operatorname{ker} \gamma=\mathfrak{a} \oplus \mathbb{R} y$ and $\operatorname{ker} \alpha=\mathfrak{a} \oplus \mathbb{R} x$, with $\gamma(x)=1$ and $\alpha(y)=1$. To see that $\mathfrak{g} / \mathfrak{a} \cong \mathfrak{a f f}$, it suffices to show that $\gamma([x, y])=0$ and $\alpha([x, y])=1$. But $\gamma([x, y])=0$ since $\operatorname{ker} \gamma$ is an ideal. And since $D \alpha=0$ one has

$$
\alpha([x, y])=d \alpha(x, y)=\gamma \wedge \alpha(x, y)=1 .
$$

Finally, notice that for all $w \in \mathfrak{g}$ one has

$$
\operatorname{tr}\left(\left.\operatorname{ad}(w)\right|_{\mathfrak{a}}\right)=\operatorname{tr}\left(\left.\operatorname{ad}(w)\right|_{\mathfrak{g}}\right)-\gamma([w, x])-\alpha([w, y])=\gamma(w)-\gamma([w, x])-\alpha([w, y]) .
$$

In particular, since $\mathfrak{a}$ is an ideal and since $\mathfrak{a} \subset \operatorname{ker} \gamma$, one has $\operatorname{tr}\left(\left.\operatorname{ad}(w)\right|_{\mathfrak{a}}\right)=0$ for all $w \in \mathfrak{a}$. Furthermore,

$$
\begin{aligned}
& \operatorname{tr}\left(\left.\operatorname{ad}(y)\right|_{\mathfrak{a}}\right)=\gamma(y)-\gamma([y, x])=0, \\
& \operatorname{tr}\left(\left.\operatorname{ad}(x)\right|_{\mathfrak{a}}\right)=\gamma(x)-\alpha([x, y])=1-\alpha([x, y])=0 .
\end{aligned}
$$

We conclude that the extension is unimodular. This proves Theorem 1.1. 


\section{REMARKS}

By Theorem 1.1, Lie algebras of cohomological codimension 1 are unimodular extensions of $\mathfrak{a} \mathfrak{f}$. Recall that an extension $\mathfrak{a} \rightarrow \mathfrak{g} \rightarrow \mathfrak{a f f}$ is inessential if $\mathfrak{g}$ possesses a subalgebra complementary to $\mathfrak{a}$. Notice that an inessential extension $\mathfrak{a} \rightarrow \mathfrak{g} \rightarrow \mathfrak{a f f}$ is completely determined by the related representation $\mathfrak{a f f} \rightarrow \operatorname{Der}(\mathfrak{a})$ of $\mathfrak{a f f}$ in the Lie algebra of derivations of degree 0 of $\mathfrak{a}$. Such representations are easy to construct and hence give examples of Lie algebras of cohomological codimension 1. We remark that the representations of $\mathfrak{a f f}$ are of two types: they are either faithful, or they are determined by a single derivation of $\mathfrak{a}$. For example, in the latter case, if $\mathfrak{a}$ is an arbitrary unimodular Lie algebra, and if $\phi$ is a trace-free derivation of $\mathfrak{a}$, then one obtains a Lie algebra $\mathfrak{g}$ of cohomological codimension 1 by introducing two additional generators $x, y$ and imposing the following relations:

$$
[x, y]=y, \quad[x, w]=\phi(w) \quad \text { for all } w \in \mathfrak{a}, \quad \text { and } \quad[y, w]=0 \quad \text { for all } w \in \mathfrak{a} .
$$

By construction, this is an inessential unfaithful unimodular extension of $\mathfrak{a f f}$.

Notice however that a given Lie algebra $\mathfrak{g}$ may be expressible in different ways as a unimodular extension of $\mathfrak{a f f}$, as the following example shows:

Example 5.1. Consider the Lie algebra $\mathfrak{g}$ of dimension 8, with $\operatorname{cd}(\mathfrak{g})=7$, having the generators $\{x, y, z, w, a, b, c, d\}$ and the following relations:

$$
\begin{aligned}
& {[x, y]=y+c, \quad[x, z]=z, \quad[x, w]=w,} \\
& {[x, a]=-a, \quad[x, c]=c, \quad[x, d]=-2 d,} \\
& {[w, a]=b, \quad[w, b]=z, \quad[w, d]=a .}
\end{aligned}
$$

Notice that $\mathfrak{g}$ is simultaneously:

(a) an essential unimodular extension of $\mathfrak{a f f}$ by the ideal $\langle w, z, a, b, c, d\rangle$,

(b) an inessential unfaithful unimodular extension of aff by $\langle w, y, a, b, c, d\rangle$,

(c) an inessential faithful unimodular extension of aff by $\langle y, z, a, b, c, d\rangle$.

We remark that if $\operatorname{dim} H^{\operatorname{dim} \mathfrak{g}-1}(\mathfrak{g}, \mathbb{R})=1$, then $\mathfrak{g}$ can be written as a unimodular extension in only one way.

Proposition. Suppose that $\mathfrak{g}$ is a finite dimensional Lie algebra with $c d(\mathfrak{g})=$ $\operatorname{dim} \mathfrak{g}-1$ and $\operatorname{dim} H^{\operatorname{dim} \mathfrak{g}-1}(\mathfrak{g}, \mathbb{R})=1$. If $\pi_{1}: \mathfrak{g} \rightarrow \mathfrak{a f f}$ and $\pi_{2}: \mathfrak{g} \rightarrow \mathfrak{a f f}$ are two unimodular extensions, then $\operatorname{ker} \pi_{1}=\operatorname{ker} \pi_{2}$.

Proof. As we saw in the proof of Theorem 1.1, the unimodular extensions $\mathfrak{g} \rightarrow \mathfrak{a f f}$ are parameterized by $H_{D}^{1}(\mathfrak{g}, \mathbb{R})$. So the Proposition follows from Theorem 2.1.

Finally, we give an equivalent alternative presentation of Theorem 1.1. First note that every finite dimensional Lie algebra $\mathfrak{g}$ is isomorphic to a codimension 1 subalgebra of a unimodular Lie algebra $\hat{\mathfrak{g}}$. Indeed, if $\mathfrak{g}$ is unimodular, we just take $\hat{\mathfrak{g}}=\mathfrak{g} \oplus \mathbb{R}$. Otherwise, let $\mathfrak{u}$ be the kernel of the canonical 1-form $\gamma$, and choose $x \in \mathfrak{g} \backslash \mathfrak{u}$ such that $\gamma(x)=1$. Then one obtains the desired algebra $\hat{\mathfrak{g}}$ by introducing an additional generator $z$ and imposing the following relations:

$$
[z, x]=z \quad \text { and } \quad[z, w]=0 \quad \text { for all } w \in \mathfrak{u} .
$$

Notice, in particular, that if $\mathfrak{m}$ denotes the Lie algebra of the group of isometries of the Minkowski plane,

$$
\mathfrak{m}=\langle x, y, z \mid[x, y]=y,[x, z]=-z\rangle,
$$


then $\mathfrak{a f f}$ is isomorphic to the subalgebra $\mathfrak{t}=\langle x, y\rangle$. More generally, Theorem 1.1 gives:

Corollary. Let $\mathfrak{g}$ be a finite dimensional Lie algebra. Then $c d(\mathfrak{g})=\operatorname{dim} \mathfrak{g}-1$ if and only if there exists a finite dimensional unimodular Lie algebra $\hat{\mathfrak{g}}$ and an epimorphism $\pi: \hat{\mathfrak{g}} \rightarrow \mathfrak{m}$ such that $\mathfrak{g}$ is isomorphic to $\pi^{-1}(\mathfrak{t})$.

Proof. One direction is immediate from Theorem 1.1. Conversely, suppose that $c d(\mathfrak{g})=\operatorname{dim} \mathfrak{g}-1$. By Theorem 1.1, $\mathfrak{g}$ is a unimodular extension of a codimension 2 ideal $\mathfrak{a}$; so we may assume that there are elements $x, y \in \mathfrak{g} \backslash \mathfrak{a}$ such that:

(a) $\mathfrak{u}=\mathfrak{a} \oplus \mathbb{R} y$ is a unimodular ideal.

(b) $[x, y]=y(\bmod \mathfrak{a})$.

Now $\mathfrak{g}$ is isomorphic to a codimension 1 subalgebra of the unimodular Lie algebra $\hat{\mathfrak{g}}$ constructed above. Furthermore, $\mathfrak{a}$ is an ideal of $\hat{\mathfrak{g}}$ and $\mathfrak{g} / \mathfrak{a} \cong \mathfrak{m}$.

\section{REFERENCES}

1. J-J. Koszul, Homologie et cohomologie des algèbres de Lie, Bull. Soc. math. France 78 (1950), 65-127. MR 12:120g

2. J. Milnor, Curvatures of left invariant metrics on Lie groups, Adv. in Math. 21 (1976), 293-329. MR 54:12970

3. H. Tasaki and M. Umehara, An invariant on 3-dimensional Lie algebras, Proc. Amer. Math. Soc. 115 (1992), 293-294. MR 92i:17009

School of Mathematics, la Trobe University, Melbourne, Australia 3083

E-mail address: matgfa@lure.latrobe.edu.au

E-mail address: G.Cairns@latrobe.edu.au

E-mail address: G.Kim@latrobe.edu.au 